\title{
Consumo de alcohol y autoestima en adolescentes
}

\author{
Alicia Álvarez Aguirre ${ }^{1}$ \\ María Magdalena Alonso Castillo² \\ Ana Carolina Guidorizzi Zanetti ${ }^{3}$
}

La presente investigación tuvo como objetivo analizar la relación entre el nivel de autoestima y el consumo de alcohol en los adolescentes, a través de la realización de un estudio descriptivo transversal, en una facultad de enfermería en la ciudad de Querétaro, en México, en el mes de julio de 2008, en una muestra de 109 adolescentes, entre 17 y 20 años. Para la obtención de los datos fueron aplicados dos instrumentos: AUDIT y Cuestionario de autoestima de Rosemberg. La mayoría de los adolescentes participantes tuvieron la autoestima elevada (94.5\%), ninguno presentó autoestima baja. El 80.7\% de los adolescentes del estudio no tiene riesgo de consumo de alcohol. Se concluye que la mayoría de los adolescentes presentaron una elevada autoestima y tuvieron riesgo bajo de consumo de alcohol. Por lo que se sugiere implementar programas preventivos con respecto al consumo de alcohol y factores protectores a fin de que los adolescentes continúen con un estilo de vida saludable.

Descriptores: Alcoholismo; Adolescente.

\footnotetext{
${ }^{1}$ Estudiante de Doctorado, Programa Doctorado en Ciencias de Enfermería, Facultad de Enfermería, Universidad Autónoma de Nuevo León, México. Profesor de Tiempo Completo, Facultad de Enfermería, Univesidad Autónoma de Querétaro, México. E-mail: alvarez_ ali@yahoo.com.mx.

2 Doctora en Filosofía con especialidad en Psicología, Profesor de Tiempo Completo, Facultad de Enfermería, Universidad Autónoma de Nuevo León, México. E-mail: magdalena_alonso@hotmail.com.

${ }^{3}$ Enfermera, Maestria em Enfermería, Escola de Enfermagem de Ribeirão Preto, Universidade de São Paulo, Centro Colaborador de la OMS para el Desarrollo de la Investigación en Enfermería, SP, Brasil. E-mail: carolzan@eerp.usp.br.
}

Correspondencia:

Ana Carolina Guidorizzi Zanetti

Universidade de São Paulo. Escola de Enfermagem de Ribeirão Preto

Av. Bandeirantes, 3900

Bairro Monte Alegre

CEP: 14040-902 Ribeirão Preto, SP, Brasil

E-mail: carolzan@eerp.usp.br 


\section{Consumo de álcool e autoestima em adolescentes}

O presente estudo teve como objetivo analisar a relação entre o nível de autoestima e o consumo de álcool em adolescentes, através da realização de estudo descritivo transversal, em uma faculdade de enfermagem de Queretaro, México, no mês de julho de 2008, com amostra de 109 adolescentes, entre 17 e 20 anos. Para obtenção dos dados, foram aplicados dois instrumentos, o AUDIT e o questionário de autoestima de Rosemberg. A maioría dos adolescentes participantes tinha autoestima elevada $(94,5 \%)$, nenhum apresentou baixa autoestima. Nesta amostra, $80,7 \%$ dos adolescentes não possui risco para consumo de álcool. Conclui-se que os adolescentes apresentaram elevada autoestima e baixo risco para o consumo de álcool. Portanto, é necessário implementar programas preventivos relacionados ao consumo de álcool e identificar os fatores protetores para garantir a manutenção de hábitos saudáveis pelos adolescentes.

Descritores: Alcoolismo; Adolescente.

\section{Alcohol Consumption and Self Esteem in Adolescents}

The aim of this study was to analyze the relationship of levels of self esteem and alcohol consumption in adolescents, by carrying out a transversal, descriptive study, in a college of nursing of Queretaro in Mexico, in the month of July 2008, with a sample of 109 adolescents, between 17 and 20 years old. For attainment of the data two instruments were applied: AUDIT and the Rosemberg self esteem scale. The majority of the participating adolescents had high self esteem (94.5\%) and none presented low self esteem. Of the adolescents in the study $80.7 \%$ did not consume alcohol hazardously. It was concluded that the adolescents presented high self esteem and low alcohol consumption. Therefore, it is necessary to implement preventive programs related to alcohol consumption and to identify the protective factors to guarantee the maintenance of healthy habits for the adolescents.

Descriptors: Alcoholism; Adolescent.

\section{Introducción}

El uso y abuso de sustancias adictivas como el alcohol, constituyen en la actualidad un fenómeno complejo, que tiene consecuencias en la salud de los individuos y su entorno, además de representar un problema sanitario en el ámbito internacional, nacional y local. Sus raíces involucran factores determinantes y condicionantes culturales, además de representar un problema global ${ }^{(1-3)}$.

En el caso de México, se informó en 2002, que cerca de tres millones de adolescentes entre 12 y 17 años consumieron una copa completa de bebida con alcohol en el año previo al estudio y de ellos las mujeres consumieron de 1 a 2 copas; las consecuencias del abuso reportadas con mayor frecuencia fueron problemas envolviendo a la policía. Además informan incremento en el índice de consumo entre los adolescentes, de $27 \%$ en 1998 a $35 \%$ en 2002 , en el género masculino y de $18 \%$ a $25 \%$ en el género femenino durante el mismo

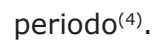

Los jóvenes son particularmente vulnerables a sufrir daños en su desarrollo e integridad cuando consumen drogas y alcohol. La adicción a estas sustancias generalmente comienza con el uso experimental y recreativo en edades tempranas. Por ello, es sumamente importante elaborar políticas con enfoque preventivo en torno a la prevención del uso y abuso de drogas y alcohol que se traduzcan en programas y proyectos que incluyan los ámbitos familiar, laboral, comunitario, escolar y a los medios de comunicación ${ }^{(5)}$.

En este proyecto preventivo, los profesionales de enfermería juegan un papel muy importante, están capacitados para realizar diagnósticos de salud, participar 
en la elaboración e implementación de programas de prevención universal, selectiva e indicativa, entre otras actividades. La enfermería necesita incorporar a su formación y práctica modelos que le permita incursionar como protagonista de la salud, específicamente en la promoción y prevención de la salud.

En el proyecto de la Comisión Interamericana para el Control del Abuso de Drogas (CICAD) de las escuelas de Enfermería sobre la Prevención del uso y abuso de las drogas y alcohol, Integración Social y Promoción de la Salud en América Latina, se menciona que el profesional de enfermería es un agente clave en el área de la salud por el cambio y la transformación que pueden producir en el individuo, familia y comunidad(6). Además, en la actualidad cumple una función esencial en el diseño y ejecución de programas de promoción de salud y prevención en varios campos interdisciplinarios, debido a que estos son los que tienen mayor contacto con las comunidades y están en condiciones de percibir la existencia de un problema como es el caso del uso y abuso de drogas y alcohol.

Los profesionales de enfermería al participar en las escuelas de educación básica deben, realizar diagnósticos de salud que les permitan conocer los factores de riesgo de la población para intervenir, de tal forma, que en base a los resultados se puedan desarrollar o potencializar los factores de protección y tener como resultado una conducta promotora de salud.

Uno de los factores de protección estudiado es la autoestima que contribuye a preservar el propio bienestar biológico, psicológico y social. El tener una autoestima saludable es considerado como un factor protector en la vida y es indispensable en la adolescencia, ya que el tener una autoestima baja, puede convertirse en factor de riesgo para iniciar el abuso de bebidas alcohólicas ${ }^{(7)}$.

La presente investigación se enfoca en identificar la relación entre el nivel de autoestima y el consumo de alcohol en una población de adolescentes mexicanos. Esto debido a que el alcohol es una sustancia psicoactiva permitida en nuestra sociedad además de que diferentes estudios de investigación han evidenciado que el alcohol es una droga de entrada al consumo de otro tipo de drogas.

\section{Metodología}

Es un estudio de enfoque cuantitativo, de tipo descriptivo y transversal, ya que los sujetos fueron evaluados una única vez. Los estudios transversales presentan ventajas en relación a su rapidez, el bajo costo, la identificación de casos y la detección de riesgo(8). En este estudio el propósito fue analizar el nivel de autoestima y el consumo de alcohol en los adolescentes.

La colecta de datos se llevó a cabo en una semana de julio de 2008, en una escuela pública de Querétaro, México. La población de estudio fue de adolescentes aspirantes a uno de los programa de licenciatura $(\mathrm{N}=300)$, la muestra se determinó por muestreo probabilístico aleatorio simple $y$, el tamaño $(n=109)$, se calculó para poblaciones finitas con un nivel de confianza de $90 \%$, probabilidad a favor de 0,80 ; probabilidad en contra de 0,20 y error de estimación de 0,05.

Los criterios de selección enunciados para este estudio fueron: todos los adolescentes entre 17 y 20 años de edad, que aspiraban ingresar a los programas de una facultad de una Universidad pública, que aceptaron participar y que se encontraban en el momento de la recolección de datos.

Antes de la recolección de datos, los participantes informaron su edad y sexo; para la recolección de datos se utilizaron dos instrumentos: Cuestionario de Identificación de los Trastornos Debidos al Consumo de Alcohol (AUDIT) y la escala de Autoestima de Rosenberg.

El AUDIT es un examen didáctico que se aplica para detectar el consumo excesivo de alcohol e identifica si el informante presenta un consumo de riesgo, un consumo perjudicial o tiene dependencia de alcohol. Esta prueba comprenden 10 preguntas que tienen una escala de puntuación de 0 a 4 puntos, el puntaje total se interpreta de la siguiente manera: 0 a 3 sin riesgo, de 4 a 7 empieza a tener problemas y 8 o más significa riesgo elevado (NOM-028-SSA2-1999).

La escala de autoestima de Rosenberg, está integrada por 10 preguntas con una escala de 1 a 4 puntos, los rangos para determinar el nivel de autoestima son: de 30 a 40 puntos: autoestima elevada, considerada como nivel de autoestima normal; de 26 a 29 puntos: autoestima promedio, este nivel indica que no se presentan problemas de autoestima grave, sin embargo conveniente mejorarla, y menos de 25 puntos: autoestima baja, en este rango existen problemas de autoestima significativos ${ }^{(9)}$.

En la recolección de los datos participaron la autora y dos investigadores colaboradores, se solicitó autorización a la institución, previa presentación del proyecto; una vez otorgado el permiso fue proporcionada una relación de los estudiantes, por control escolar, aspirantes al programa de licenciatura. 
Por medio de carteles e invitación personal en la sala de clases, se convocó a todos los aspirantes a participar en el estudio, a los que se mostraron interesados se los citó a la escuela de estudio, en una sala privada para la firma de su consentimiento y aplicación de los dos instrumentos, lo llevó en promedio 20 minutos; la entregar los instrumentos se verificó que estuvieran contestadas todas las preguntas.

Para el tratamiento de la información se realizó una base de datos que incluía cada una de las interrogantes del instrumento en el programa estadístico para las ciencias sociales - SPSS, versión 12 . Se procedió a revisar la consistencia interna de cada uno de los instrumentos a través de alpha de Crombach. Se utilizó estadística descriptiva: frecuencias y porcentajes en las variables: sexo, rangos de autoestima y consumo de alcohol; valor mínimo y máximo, promedio, mediana y desviación estándar en la variable edad; los resultados se presentaron en tablas.

La presente investigación fue sometida al comité de ética de la Facultad de Enfermería de la Universidad
Autónoma de Querétaro con aprobación en 14 de febrero de 2008.

\section{Resultados}

De los 109 adolescentes entrevistados 48 (44\%) eran de género masculino y 61 (56\%) de género femenino. La edad de las personas participantes en el estudio varió entre 17 y 20 años ( $D E=1,013)$.

De acuerdo con la escala de autoestima que se muestra en la Tabla 1, los informantes están totalmente de acuerdo en: 1) ser una persona de igual valor que otras personas $(92,7 \%), 2)$ tiene muchas buenas cualidades $(67,7 \%), 3)$ puede hacer las cosas igual que Ios demás $(54,1 \%)$, y 4 ) tiene una actitud positiva con su persona (76,1\%); estuvieron de acuerdo en: 1 ) sentirse satisfechos consigo mismo $(48,6 \%), 2)$ desearían tener más respeto por sí mismo (35.8\%); indicaron estar totalmente en desacuerdo en: 1) creer que son un fracaso $(87.2 \%), 2)$ no tiene mucho porque sentirse orgulloso $(54.8 \%), 3$ ) en sentirse inútiles $(56.0 \%)$, y 4$)$ en creer que no sirven para nada $(74.3 \%)$.

Tabla 1 - Frecuencias de Autoestima de los participantes (Querétaro, México 2008)

\begin{tabular}{|c|c|c|c|c|}
\hline Variable & $\begin{array}{c}\text { Totalmente de } \\
\text { acuerdo }\end{array}$ & De acuerdo & En desacuerdo & $\begin{array}{c}\text { Totalmente en } \\
\text { desacuerdo }\end{array}$ \\
\hline & $\mathbf{F}(\%)$ & $F(\%)$ & $F(\%)$ & $F(\%)$ \\
\hline 1. Siento que soy una persona de valor, al igual que otras personas & $101(92.7)$ & $8(7.3)$ & $0(0)$ & $0(0)$ \\
\hline 2. Definitivamente creo que soy un fracaso & $1(0.9)$ & $0(0)$ & $13(11.9)$ & $95(87.2)$ \\
\hline 3. Siento que tengo muchas buenas cualidades & $73(67.7)$ & $34(31.2)$ & $1(0.9)$ & $1(0.9)$ \\
\hline 4. Puedo hacer las cosas igual que los demás & $59(54.1)$ & $42(38.5)$ & $6(5.5)$ & $2(1.8)$ \\
\hline 5. No tengo mucho porque sentirme orgullosa & $2(1.9)$ & $12(11.0)$ & $32(29.4)$ & $63(54.8)$ \\
\hline 6. Tengo una actitud positiva con mi persona & $83(76.1)$ & $26(23.9)$ & $0(0)$ & $0(0)$ \\
\hline 7. En todo, estoy satisfecha conmigo misma & $50(45.9)$ & $53(48.6)$ & $4(3.7)$ & $2(1.8)$ \\
\hline 8. Desearía tener más respeto conmigo misma & $30(27.5)$ & $39(35.8)$ & $17(15.6)$ & $23(22.1)$ \\
\hline 9. Algunas veces me siento inútil & $0(0)$ & $17(15.6)$ & $31(28.4)$ & $61(56.0)$ \\
\hline 10. Algunas veces, creo que no sirvo para nada & $0(0)$ & $11(10.1)$ & $17(15.6)$ & $81(74.3)$ \\
\hline
\end{tabular}

Fuente: Escala de Rosenberg

$\mathrm{n}=109$

De acuerdo con los rangos de la escala de autoestima, Tabla2, que presentaron los participantes, como se muestra en la Tabla 1 , se observa que el $94,5 \%$ presenta autoestima elevada, 5,5\% promedio y no hay presencia de autoestima baja.

Tabla 2 - Rangos de Autoestima (Querétaro, México 2008)

\begin{tabular}{lc}
\hline \multicolumn{1}{c}{ Rangos } & Frecuencia (\%) \\
\hline Autoestima Elevada (30-40 puntos) & $103(94,5)$ \\
Autoestima promedio (26-29 puntos) & $6(5,5)$ \\
Autoestima baja (menos de 25 puntos) & $0(0,0)$ \\
\hline
\end{tabular}

Fuente: Escala de Rosenberg

$\mathrm{n}=109$
En la Tabla 3, se aprecia que un $44 \%$ consume al mes, entre una o menos veces, alguna bebida alcohólica, el $17,4 \%$ de los participantes suele consumir de 3 a 4 bebidas alcohólicas en un día normal, en tanto que el $15,6 \%$ consume 6 o más bebidas alcohólicas en un solo día; en el curso del último año el 3,7\% ha sido incapaz de dejar de beber una vez que había empezado a consumir, 2,8\% no pudo hacer lo que se esperaba de él porque había bebido, el $2.8 \%$ ha necesitado beber en ayunas para recuperarse después de haber bebido mucho el día anterior, el 9,2\% ha tenido remordimiento o sentimientos de culpa después de haber bebido, menos de una vez al mes, el $0,9 \%$ no ha podido recordar lo 
que sucedió la noche anterior porque había bebido, el $100 \%$ menciona no haber resultado herido, el mismo u otra persona, por haber bebido, el 3,7\% indica que algún familiar, amigo, médico o profesional sanitario ha mostrado preocupación por su consumo de bebidas alcohólicas o le ha sugerido que deje de beber.

Tabla 3 - Frecuencias de Consumo de alcohol de los participantes (Querétaro, México 2008)

\begin{tabular}{|c|c|c|c|c|c|}
\hline Variable & O F ( $\%)$ & $1 \mathrm{~F}(\%)$ & $2 \mathrm{~F}(\%)$ & 3 F (\%) & $4 \mathrm{~F}(\%)$ \\
\hline ¿Con que frecuencia consume alguna bebida alcohólica? & $43(39,4)$ & $48(44)$ & $17(15,6)$ & $1(0,9)$ & $0(0)$ \\
\hline ¿Cuántas bebidas alcohólicas suele realizar en un día de consumo normal? & $82(75,2)$ & $19(17,4)$ & $8(7,3)$ & $0(0)$ & $0(0)$ \\
\hline ¿Con que frecuencia toma 6 o más bebidas alcohólicas en un solo día? & $85(78)$ & $17(15,6)$ & $7(6,4)$ & $0(0)$ & $0(0)$ \\
\hline $\begin{array}{l}\text { ¿Con que frecuencia en el curso del último año ha sido incapaz de beber una vez que había } \\
\text { empezado? }\end{array}$ & $102(93,6)$ & $4(3,7)$ & $1(0,9)$ & $1(0,9)$ & $1(0,9)$ \\
\hline $\begin{array}{l}\text { ¿Con que frecuencia en el curso del último año no pudo hacer lo que se esperaba de usted } \\
\text { porque había bebido? }\end{array}$ & $105(96,3)$ & $3(2,8)$ & $1(0,9)$ & $0(0)$ & $0(0)$ \\
\hline $\begin{array}{l}\text { ¿Con que frecuencia en el último año ha necesitado beber en ayunas para recuperarse } \\
\text { después de haber bebido mucho el día anterior? }\end{array}$ & $106(97,2)$ & $3(2,8)$ & $0(0)$ & $0(0)$ & $0(0)$ \\
\hline $\begin{array}{l}\text { ¿Con que frecuencia en el curso del último año ha tenido remordimiento o sentimientos de } \\
\text { culpa después de haber bebido? }\end{array}$ & $98(89,9)$ & $10(9,2)$ & $1(0,9)$ & $0(0)$ & $0(0)$ \\
\hline $\begin{array}{l}\text { ¿Con que frecuencia el curso del último año no ha podido recordar lo que sucedió la noche } \\
\text { anterior por que había estado bebiendo? }\end{array}$ & $108(99,1)$ & $1(0,9)$ & $0(0)$ & $0(0)$ & $0(0)$ \\
\hline ¿Usted o alguna otra persona ha resultado herido porque usted había bebido? & $109(100)$ & $0(0)$ & $0(0)$ & $0(0)$ & $0(0)$ \\
\hline $\begin{array}{l}\text { ¿Algún familiar, amigo, médico o profesional sanitario ha mostrado preocupación por su } \\
\text { consumo de bebidas alcohólicas o le ha sugerido que deje de beber? }\end{array}$ & $103(94,5)$ & $0(0)$ & $4(3,7)$ & $0(0)$ & $2(1,8)$ \\
\hline
\end{tabular}

Fuente: Cuestionario AUDIT $n=109$

En la Tabla 4, se observan los dominios de consumo de alcohol en los que se encontraban los participantes, $80,7 \%$ sin riesgo, $13,8 \%$ empiezan a tener problemas y $3,7 \%$ presentó riesgo elevado.

Tabla 4 - Consumo de alcohol en la población participante (Querétaro, México 2008)

\begin{tabular}{lc}
\hline \multicolumn{1}{c}{ Variable } & Frecuencia (\%) \\
\hline Sin riesgo & $88(80,7)$ \\
Empieza a tener problemas & $15(13,8)$ \\
Riesgo elevado & $4(3,7)$ \\
\hline
\end{tabular}

Fuente: Cuestionario AUDIT

$n=109$

De acuerdo con la Tabla 5, la mayoría de los adolescentes posee autoestima elevada y sin riesgo para el consumo de alcohol ( $81 \%)$, el $14 \%$ posee autoestima promedio y empieza a tener riesgo y el $5 \%$ presenta autoestima baja y riesgo elevado. Por tanto, de acuerdo con los resultados de este estudio, cuando el adolescente presenta autoestima elevada menor es el riesgo de consumir alcohol.
Tabla 5 - Nivel de autoestima y consumo de alcohol (Querétaro, México 2008)

\begin{tabular}{lcccc}
\hline \multirow{2}{*}{ Variable } & \multicolumn{3}{c}{ AUDIT } & \multirow{2}{*}{ Total } \\
\cline { 2 - 4 } & $\begin{array}{c}\text { Sin } \\
\text { riesgo }\end{array}$ & $\begin{array}{c}\text { Empieza a } \\
\text { tener riesgo }\end{array}$ & $\begin{array}{c}\text { Riesgo } \\
\text { elevado }\end{array}$ \\
\hline Autoestima elevada & $84(81 \%)$ & $15(14 \%)$ & $4(5 \%)$ & $103(100 \%)$ \\
Autoestima promedio & $5(83 \%)$ & $1(17 \%)$ & $0(0 \%)$ & $6(100 \%)$ \\
Total & $89(82 \%)$ & $16(15 \%)$ & $4(3 \%)$ & $109(100 \%)$ \\
\hline
\end{tabular}

Fuente: Cuestionario AUDIT

$\mathrm{n}=109$

\section{Discusión}

La mayoría de los adolescentes fueron del sexo femenino con edad promedio de 18 años, la mayoría de los participantes tuvieron autoestima elevada 94,5\%, ninguno presentó autoestima baja. Lo anterior hacer referencia a que la autoestima de los adolescentes es bastante estable a corto plazo, no así a lo largo de varios años ${ }^{(10)}$

El $80,7 \%$ de los adolescentes del estudio no tiene riesgo de consumo de alcohol, esta condición se describe en la literatura como la de individuos que poseyendo un mayor nivel de autoestima presentan mayores 
mecanismos de defensa ante el consumo de alcohol(1). Este punto coincide con los postulados de un autor que define la autoestima como la resultante perceptiva emotiva de la autoevaluación al interaccionar con otros poniendo a prueba la autoeficacia(11).

Estos resultados corroboran los hallazgos de los estudios, que encontraron que una baja autoestima constituye un factor de riesgo para el consumo de alcohol, es decir cuando la autoestima se encuentra amenazada por un evento negativo, se produce un incremento en los niveles de ansiedad, el individuo reacciona buscando otras alternativas para enfrentar la situación, en muchos casos se derivan para formas poco apropiadas o perjudiciales para la salud, como es el caso del consumo de alcohol; ante estos resultados los autores sugieren prestar atención al nivel de autoestima en cuanto a la prevención del consumo de alcohol en esta población, con atención especial a las diferencias de género ${ }^{(12-15)}$.

Así este estudio está de acuerdo con lo informado por otros autores, al referirse a la autoestima como un factor que disminuye la probabilidad del consumo de drogas. Así, un buen nivel de autoestima es considerado un factor protector que aísla al sujeto de las influencias no saludables, ya que estas personas tienen una menor vulnerabilidad ante la conducta antisocial(16-21). Entonces, se reafirma que la autoestima es un recurso psicológico que fomenta el ajuste saludable del adolescente(18).
Por lo tanto, estos resultados proporcionan bases para plantear estrategias que favorezcan las conductas promotoras de los adolescentes.

\section{Conclusión}

Podemos concluir que los adolescentes presentaron una elevada autoestima y tuvieron riesgo bajo de consumo de alcohol. Por lo que se sugiere implementar programas preventivos con respecto al consumo de alcohol y factores protectores a fin de que los adolescentes continúen con un estilo de vida saludable.

\section{Agradecimientos}

Agradecemos a la Comisión Interamericana para el Control del Abuso de Drogas/CICAD de la Secretaria de Seguridad Multidimensional/SSM de la Organización de los Estados Americanos/OEA, la Secretaria Nacional de Políticas sobre Drogas/SENAD do Gabinete de Seguridad Institucional/Brasil, la Escuela de Enfermería de Ribeirao Preto de la Universidad de Sao Paulo y Centro Colaborador de la Organización Mundial de la Salud para el Desarrollo de la Investigación en Enfermería, la población representada en los estudios de investigación, bien como a las autoridades de las universidades representadas por los participantes del Programa En-Line de Especialización en Investigación sobre el Fenómeno de las Drogas PREINVEST, periodos 2005, 2006, 2007 y 2008.

\section{Referencias}

1. Musayón Y, Torres C, Sánchez E, Chavez E. Factores de riesgo del consume de bebidas alcoholicas en escolares de educación secundaria. Invest Educ Enferm. 2005; 23(1):54-67.

2. Newcomb M. Identifying high-risk youth:prevalence and patterns of adolescent drug abuse. En: Rahdert E, Czechwicz D, editors. Adolescent drug abuse: clinical assessment and therapeutic interventions. Rockville, Maryland: National Institute on Drug abuse; 1995. p. 7-37. (NIDA Res Monogr Ser No 156) 3. Murray $D$, Perry $C$. The prevention of adolescent drug abuse: implications of etiological, developmental, behavioral, and environmental models. En: Jones $\mathrm{C}$, Battjes R, editors. Etiology of drug abuse: implications for prevention. Rockville, Maryland: National Institute on Drug Abuse; 1995. p. 236-56. (NIDA Res Monoger Ser No 56)

4. Secretaria de Salud Dirección General de Epidemiología (MX). Encuesta Nacional de Adicciones (ENA-2002, ENA-2008) México; 2008.

5. Díaz Negrete B, García-Aurrecoechea R. Factores psicosociales de riesgo de consumo de drogas ilícitas en una muestra de estudiantes mexicanos de educación media. Rev Panam Salud Publica. 2008; 24(4):223-32.
6. Comisión Interamericana para el Control del Abuso de Drogas. Escuelas de enfermería sobre la prevención del uso y abuso de las drogas, integración social y promoción de la salud en América Latina. Washington: OEA/OPS; 2001.

7. Centros de Integración Juvenil Como proteger a tus hijos contra las drogas. México: CIJ; 1999.

8. Pereira MG. Epidemiologia: Teoria e prática. Rio de Janeiro: Ed. Guanabara Koogan; 1995.

9. Pereira NBR, Duvicq CGF, Carvalho AMP. Influencia del consumo de sustancias psicoactivas en el ámbito familiar sobre la autoestima de escolares. Rev. Latino-Am. Enfermagem. 2005; 13(num esp):798-805.

10. Rosenberg Society and the adolescent self-image (Revised edition). Middletown: Wesleyan University; 1989

11. Ramírez A. La sociedad y tú, todo un reto. Comunicación estrés y autoestima. Salamanca (ES): Amarrú Edicciones; 1997. 12. Chávez LMC, Andrade D. La escuela en la prevención del consumo de alcohol y tabaco: retrato de una realidad. Rev. Latino-Am. Enfermagem. 2005; 13 (núm esp):1-10. 
13. Martínez Maldonado R, Pedrão LJ, Alonso Castillo MM, López García KS, Oliva Rodríguez NN. Autoestima, autoeficacia percibida, consumo de tabaco y alcohol en estudiantes de educación secundaria de área urbana y rural de Monterrey, Nuevo León, México. Rev. Latino-Am. Enfermagem. julho-agosto 2008; 16(número especial):614-20.

14. López GT, Moreno JB. La Depresión y Autoestima como Predictores de Comportamientos de altos riesgos. Madrid: Universidad Autónoma de Madrid; 2002.

15. Engels RCME, Hale III WW, Noom M, Vries H. Self-efficacy and emotional adjustment as precursors of smoking in early adolescence. Subst Use Misuse. 2005; 40:1883-93.

16. Alonso-Castillo MM, Guzmán-Facundo FR, López-García KS, Trujillo-Martínez MP. Afecto y practica de estilos de vida en adolescentes. Desarroll Cient Enferm. 2001; 9(10):291-6.
17. Brendgen M, Vitaro F, Turgeon L, Poulin F, Wanner B. Is there a dark side of positive illusions? Overestimation of social competence and subsequent adjustment in aggressive and nonaggressive children. Abnorm Psychol. 2004; 32(3):305-20.

18. Musitu G, Jiménez TI, Murgui S. Funcionamiento familiar, autoestima y consumo de sustancias en adolescentes: un modelo de mediación. Salud Pública México. 2007; 49(1):3-10.

19. Navarro M, Pontillo V. Autoestima del adolescente y riesgo de consumo de alcohol. Actual Enferm. 2002; 5(1):7-12.

20. Armendáriz García NA, Rodríguez Aguilar L, Guzmán Facundo FR. Efecto de la autoestima sobre el consumo de tabaco y alcohol en adolescentes del área rural de Nuevo León, México. SMAD Rev Electr Salud Mental Alcohol y Drogas. 2008; 4(1):1-15.

21. Becoña I. Factores de riesgo y Protección. Bases teóricas que sustentan los programas de prevención de drogas: Plan Nacional de drogas. España: Universidad de Santiago de Compostela; 1999. 